\title{
A Joint Crest Factor Reduction and Digital Predistortion for Power Amplifiers Linearization Based on Clipping-and-Bank-Filtering
}

\author{
Siqi Wang, Morgan Roger, Member, IEEE, Julien Sarrazin, Member, IEEE, and Caroline Lelandais-Perrault
}

\begin{abstract}
The power efficiency and the linearity of a power amplifier (PA) depend on its operating point. A high efficiency generally corresponds to poor linearity. To optimize the efficiency/linearity trade-off, crest factor reduction (CFR) techniques are classically implemented along with digital predistortion (DPD) to control the PA operating point. Joint CFR/DPD can be realized with a single model so that the running complexity of the CFR is negligible. This paper fully explores the clipping-andbank-filtering (CABF) method for joint CFR/DPD and extends it to the multi-carrier case with validation on experimental results. Compared with conventional approaches, it provides either better linearization performance or lower complexity at the same PA operating point. The study is completed with a discussion on the choice of windows in the filter bank. The proposed CABF-based joint CFR/DPD model is then experimentally evaluated on a test bench with two PAs using single-carrier and 2-carrier $20 \mathrm{MHz}$ long term evolution (LTE) signals as the stimulus.
\end{abstract}

Index Terms-Crest factor reduction, digital predistortion, nonlinear distortion, power amplifiers, running complexity

\section{INTRODUCTION}

$\mathbf{I}$ N MODERN wireless telecommunication systems, power amplifiers (PA) consume the majority of power. Improving their power efficiency in transmitting data is an important concern for green communications [1]. The power efficiency of the PA is strongly correlated to its operating point [2]. When the PA operates near the saturation zone, the power efficiency approaches its maximum but the PA is very nonlinear.

Digital predistortion (DPD) is an efficient choice to compensate for the nonlinearities and the memory effects of the PA near saturation. Basically the DPD has the inverse characteristics of the PA and is applied upstream of the PA in a transmitter circuit [3]. Numerous DPD models based on Volterra series have been studied, such as memory polynomial (MP) [4] [5], generalized memory polynomial (GMP) [6], dynamic-deviation-reduction (DDR) [7], complexity-reduced Volterra series (CRV) [8], and the decomposed vector rotationbased behavioral model (DVR) [9]. Block-oriented non linear (BONL) systems have also been studied as DPD [10]-[12].

S. Wang, M. Roger, C. Lelandais-Perrault are with GeePs - Group of electrical engineering - Paris, CNRS, CentraleSupélec, Univ. Paris-Sud, Université Paris-Saclay, Sorbonne Université, 3 \& 11 rue Joliot-Curie, Plateau de Moulon 91192 Gif-sur-Yvette CEDEX, France (e-mail of authors: firstname.surname@centralesupelec.fr).

J. Sarrazin is with the Sorbonne Université, Laboratory of Electronics and Electromagnetism, L2E, F-75005 Paris, France (e-mail: julien.sarrazin@sorbonne-universite.fr).

Color versions of one or more of the figures in this paper are available online at http://ieeexplore.ieee.org.
With the linearization by DPD, the main constraint on the PA operating point is the peak-to-average power ratio (PAPR) of the modulated signal, e.g. Orthogonal Frequency Division Multiplexing (OFDM) signals [13]. In order to avoid the PA saturation, an output back-off (OBO) at least equal to the PAPR is needed.

Numerous crest factor reduction (CFR) techniques have been studied to reduce the PAPR when DPD is used [14][16]. CFR is usually implemented as a correction to reduce signal peaks. It can be applied at the DPD input or the DPD output — even both — with different methods [17].

Though CFR helps increasing the PA efficiency, it deteriorates the system linearity, especially if basic hard clipping (HC) is used. The error vector magnitude (EVM) and the adjacent channel power ratio (ACPR) are often used to characterize the in-band and out-of-band errors respectively. As the ACPR requirements are more stringent than that of EVM, the clipand-filter (CAF) approach enables to trade off in-band errors for reduced out-of-band errors compared to HC [18]. Another approach is to implement the CFR as a parametric model, which we refer to as modeled CFR (MCFR). For instance, a polynomial soft clipping method has been proposed in [19] as CFR at the DPD input. The polynomial coefficients are estimated by minimizing the PAPR under the constraints of ACPR requirements. Because the soft clipping compresses only the signal amplitude, the EVM value can be improved at the receiver using a post-compensation method.

In [14], the MCFR is applied on the DPD output signal. If the MCFR and the DPD share the same model structure, they can be merged into a single joint CFR/DPD model whose coefficients are equal to the difference of the MCFR and the DPD coefficients. In [17], the authors compared different CFR methods by studying their impact on linearity for several OBO values, in the presence of DPD. The joint CFR/DPD model is found having a great advantage in terms of the computational burden added by the CFR. The joint CFR/DPD by a single model has the same running complexity as the DPD. This is especially interesting in the applications of coming $5 \mathrm{G}$ and femtocell systems because they have more stringent requirements on the complexity of the digital signal processing part since this complexity is no longer negligible to the power consumed by the PA [20], [21].

Regarding model coefficient estimation, the model input signal and the its desired output signal are necessary. The DPD identification is usually based on the input and the output signals of the PA [22]. The MCFR coefficients can be 


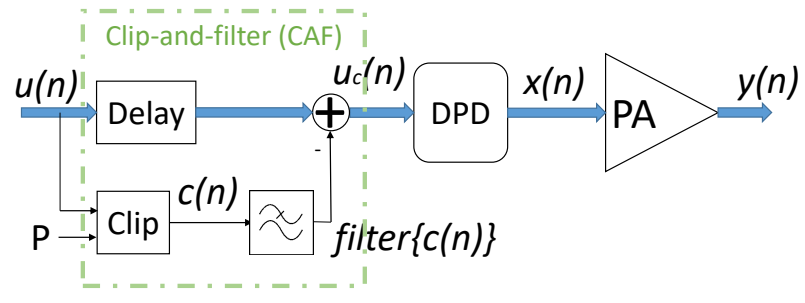

Fig. 1. The structure of CAF in front of DPD.

estimated in a similar way using the stimulus and a reference correction signal. The reference correction signal has a great impact on the performance of the joint CFR/DPD model in terms of linearity.

In the literature for joint CFR/DPD structures [14], the reference correction signal is generated by clipping the predistorted signal using CAF. In [23], a clipping-and-bankfiltering $(\mathrm{CABF})$ approach is proposed to improve the accuracy of the reference correction signal for single-carrier stimuli. Simulations showed promising results.

This paper generalizes the CABF method to multi-carrier stimulus and demonstrates its validity with experimental results. It also discusses the choice of windows for the CABF decomposition filters. The proposed method is validated on a test bench with two real PAs, first with a single-carrier then a 2-carrier $20 \mathrm{MHz}$ long term evolution (LTE) signal.

This paper is organized as follows. Section II recalls the conventional methods of implementing CFR and DPD. Section III presents the structure of the proposed CABF-based joint CFR/DPD, different approaches to compute the reference correction signal for single-carrier and multi-carrier stimuli are detailed. In Section IV, the experimental results are presented and discussed. Finally, the conclusion is given in Section V.

\section{Conventional CFR AND DPD MEthods}

\section{A. Traditional CAF with DPD}

CAF is one of the most used CFR algorithms. When used in conjunction with DPD, it is generally applied on the DPD input, as in Fig. 1, since this architecture provides better performances [17]. The clipping principle is to detect the peaks of the DPD input signal $u(n)$ with a given threshold $P$ and to compute the corresponding correction signal

$$
c(n)= \begin{cases}u(n)\left(1-\frac{P}{|u(n)|}\right) & \text { if }|u(n)| \geq P, \\ 0 & \text { otherwise, }\end{cases}
$$

This hard clipping (HC) introduces undesirable out-of-band frequency components. The CAF applies a filter to trade-off out-of-band components of $c(n)$ for in-band components. The final signal is therefore

$$
u_{c}(n)=u(n)-\text { filter }\{c(n)\} .
$$

Since filtering $c(n)$ may generate new peaks in $u_{c}(n)$, it is possible to repeat the CAF procedure for several iterations [24] and finally apply $\mathrm{HC}$ to definitely remove any remaining peak.

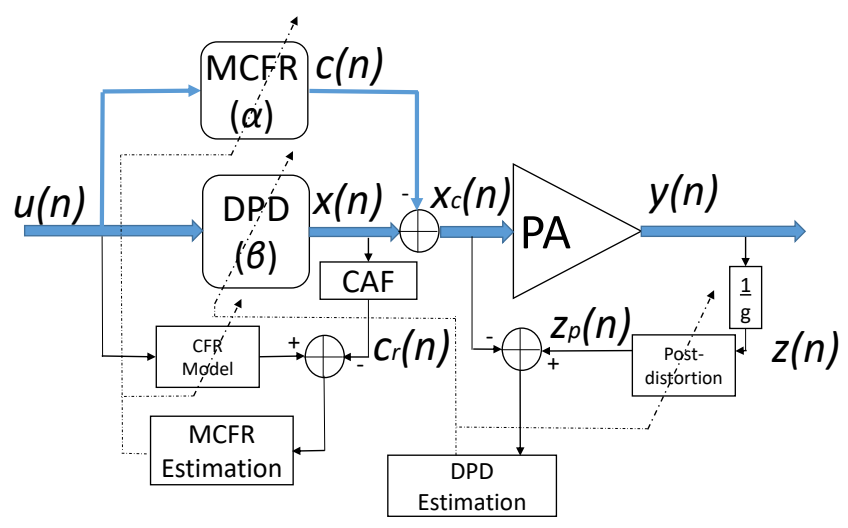

Fig. 2. Conventional joint CFR/DPD structure [14].

\section{B. Conventional joint CFR/DPD structure}

Compared with the traditional CFR method above, a joint CFR/DPD structure offers reduced running complexity if the CFR is implemented as a parametric model (MCFR) identical to the one used for the DPD, since the CFR can simply be implemented as a correction of the DPD coefficients (see Section IV). The corresponding structure is illustrated in Fig. 2. The PAPR of the DPD output signal is reduced with an MCFR block.

The DPD and the MCFR share the same model structure $\Phi$, respectively with parameters $\beta$ and $\alpha$, so that

$$
\begin{aligned}
& c(n)=\Phi_{\alpha}[u(n)] \\
& x(n)=\Phi_{\beta}[u(n)],
\end{aligned}
$$

where $u(n)$ is the system input signal or stimulus, $c(n)$ is the MCFR output and $x(n)$ is the DPD output. Thus the clipped predistorted signal $x_{c}(n)$ is

$$
x_{c}(n)=x(n)-c(n)=\Phi_{\theta}[u(n)]
$$

where $\theta=\beta-\alpha$.

Studies in [25] [26] show that the GMP model structure has good linearization performance compared with other mathematical models. Moreover, since it is linear w.r.t. its parameters, the identification can be performed simply with least squares (LS). In this paper, we use the GMP for the joint CFR/DPD model structure, which can be written as

$$
\begin{aligned}
\Phi_{\theta}[u(n)]= & \sum_{k=0}^{\mathcal{K}_{a}-1} \sum_{l=0}^{\mathcal{L}_{a}-1} \theta_{a, k l} u(n-l)|u(n-l)|^{k} \\
& +\sum_{k=1}^{\mathcal{K}_{b}} \sum_{l=0}^{\mathcal{L}_{b}-1} \sum_{m=1}^{\mathcal{M}_{b}} \theta_{b, k l m} u(n-l)|u(n-l-m)|^{k} \\
& +\sum_{k=1}^{\mathcal{K}_{c}} \sum_{l=0}^{\mathcal{L}_{c}-1} \sum_{m=1}^{\mathcal{M}_{c}} \theta_{c, k l m} u(n-l)|u(n-l+m)|^{k}
\end{aligned}
$$

where $k$ is the index for nonlinearity, and $l, m$ are the indices for memory. The vector $\theta=\left[\theta_{a}, \theta_{b}, \theta_{c}\right]$ contains the complex coefficients for the signal and envelope, the signal and lagging envelope, and the signal and leading envelope, respectively. $\mathcal{K}_{a}, \mathcal{K}_{b}, \mathcal{K}_{c}$ are the highest orders of nonlinearity. $\mathcal{L}_{a}, \mathcal{L}_{b}, \mathcal{L}_{c}$ 
are the highest memory depths. $\mathcal{M}_{b}, \mathcal{M}_{c}$ denote the longest lagging and leading delay tap lengths, respectively.

The MCFR coefficients $\alpha$ are estimated using the stimulus $u(n)$ and the reference correction signal $c_{r}(n)$ as shown in Fig. 2. This reference correction signal is obtained by applying a traditional CFR method, such as the CAF above, on the DPD output

$$
c_{r}^{h c}(n)= \begin{cases}x(n)\left(1-\frac{P}{|x(n)|}\right) & \text { if }|x(n)| \geq P, \\ 0 & \text { otherwise }\end{cases}
$$

and the conventional $\mathrm{CAF}$ with ideal rectangular window can be expressed in frequency domain as

$$
\mathcal{F}\left\{c_{r}^{c a f}\right\}= \begin{cases}\mathcal{F}\left\{c_{r}^{h c}\right\}(\omega) & \text { if } \omega \in\left[-\frac{B}{2}, \frac{B}{2}\right], \\ 0 & \text { otherwise, }\end{cases}
$$

where $B$ is the bandwidth of the stimulus $u(n)$ and $\mathcal{F}\{\cdot\}$ represents discrete Fourier Transform.

\section{PROPOSED JOINT CFR/DPD}

The structure of the proposed CABF-based joint CFR/DPD is depicted in Fig. 3, where $x_{c}(n)$ is the output signal of the joint CFR/DPD. The part with thick blue lines represents the signal transmission system of the test bench. Other parts are structures of model coefficient estimation which can be computed without interrupting the signal transmission.

\section{A. Model Identification}

The identification of the joint CFR/DPD in Fig. 3 is based on indirect learning architecture (ILA). We estimate the values of $\alpha$ and $\beta$ in an iterative procedure.

Inside each iteration, the DPD coefficients $\beta$ are estimated before the CFR coefficients $\alpha$. The first step is the identification of the post-distortion block using the PA input $x_{c}(n)$ and the signal $z(n)$ which is the PA output $y(n)$ divided by $g$, the desired loop gain. As different possible choices for $g$ achieve about the same PA efficiency [27], we choose the small signal gain in this paper.

As mentioned above, one advantage of choosing a model structure derived from Volterra series is that the model coefficients can be estimated by solving a linear problem. We can express the post-distortion using matrix notation for a block of $N$ samples:

$$
z_{p}=\mathbf{Z} \boldsymbol{\beta}
$$

where $\boldsymbol{z}_{\boldsymbol{p}}=\left[z_{p}(1), \ldots, z_{p}(N)\right]^{T}, \boldsymbol{z}=[z(1), \ldots, z(N)]^{T}, \mathbf{Z}$ is $N \times R$ matrix containing basis functions of $\boldsymbol{z}$, and $R=$ $\mathcal{K}_{a} \mathcal{L}_{a}+\mathcal{K}_{b} \mathcal{L}_{b} \mathcal{M}_{b}+\mathcal{K}_{c} \mathcal{L}_{c} \mathcal{M}_{c}$ is the total number of coefficients. The LS estimation of $\boldsymbol{\beta}$ is found by

$$
\hat{\boldsymbol{\beta}}=\left[\mathbf{Z}^{H} \mathbf{Z}\right]^{-1} \mathbf{Z}^{H} \boldsymbol{x}_{\boldsymbol{c}}
$$

which minimizes the cost function

$$
C=\sum_{n=1}^{N}\left|z_{p}(n)-x_{c}(n)\right|^{2}
$$

The identified post-distortion is then applied upstream of the PA as the DPD.
With the current estimate $\hat{\boldsymbol{\beta}}$, the CFR model coefficients $\boldsymbol{\alpha}$ can be estimated to reduce the PAPR at the DPD output by

$$
\hat{\boldsymbol{\alpha}}=\left[\mathbf{U}^{H} \mathbf{U}\right]^{-1} \mathbf{U}^{H} \boldsymbol{c}_{\boldsymbol{r}}
$$

where $\boldsymbol{U}$ is the basis function matrix of $\boldsymbol{u}$ and $\boldsymbol{c}_{\boldsymbol{r}}$ is the vector of reference correction signal.

The initial value of $\boldsymbol{\alpha}$ is an array of 0 . The initial value of $\boldsymbol{\beta}$ is $[1,0, \cdots, 0]$. Thus at the first iteration, $\boldsymbol{x}_{\boldsymbol{c}}=\boldsymbol{u}$. We repeat 4 iterations in this paper for convergence.

Since $\alpha$ is estimated according to $\boldsymbol{u}$ and $\boldsymbol{c}_{\boldsymbol{r}}$, the generation method of $\boldsymbol{c}_{\boldsymbol{r}}$ has a great impact on the system performance. Simulations previously showed that the CABF approach could outperform the conventional CAF approach in generating $\boldsymbol{c}_{\boldsymbol{r}}$ with a single-carrier stimulus [23].

\section{B. CABF-based Joint CFR/DPD with Single-Carrier Stimulus}

The reference correction signal is determined using a clipped version of the DPD output to limit the PA input under a certain threshold. The maximum PA input peak power $P_{\text {PAinPeak }}$ is constrained by its corresponding output power being the edge of the PA saturation zone as shown in Fig. 4. In the following, we denote the clipping threshold of the CFR with $P=P_{\text {PAinPeak }}$.

In this section, we consider a stimulus with only one carrier. Since the chosen DPD model is based on Volterra series, the predistorted signal $x(n)$ contains the nonlinearities brought by $|u(n)|^{k}$ according to (3) and (5). Hence the bandwidth $B$ is not appropriate for $x(n)$. We decompose $x(n)$ into signals of different nonlinearity orders as

$$
x(n)=\sum_{k \in \mathcal{K}} g_{k}(n)
$$

where

$$
\begin{aligned}
g_{k}(n)= & \sum_{l=0}^{\mathcal{L}_{a}-1} \beta_{a, k l} u(n-l)|u(n-l)|^{k-1} \\
& +\sum_{l=0}^{\mathcal{L}_{b}-1} \sum_{m=1}^{\mathcal{M}_{b}} \beta_{b, k l m} u(n-l)|u(n-l-m)|^{k-1} \\
& +\sum_{l=0}^{\mathcal{L}_{c}-1} \sum_{m=1}^{\mathcal{M}_{c}} \beta_{c, k l m} u(n-l)|u(n-l+m)|^{k-1}
\end{aligned}
$$

is the signal with nonlinearity order $k$. Since $u(n)$ is limited in $\left[-\frac{B}{2}, \frac{B}{2}\right]$, the spectrum of $g_{k}(n)$ occupies the band $\left[-k \frac{B}{2}, k \frac{B}{2}\right]$. In order to keep the nonlinearity information in $x(n)$, we clip and filter each $g_{k}(n)$ separately within corresponding bands.

As illustrated in Fig. 3, first we detect the peaks of $x(n)$ to get the clipped signal $d_{k}(n)$ corresponding to each $g_{k}(n)$ :

$$
d_{k}(n)= \begin{cases}g_{k}(n)\left(1-\frac{P}{|x(n)|}\right) & \text { if }|x(n)| \geq P, \\ 0 & \text { otherwise. }\end{cases}
$$

Then $d_{k}(n)$ is filtered according to its nonlinearity order $k$ :

$$
\mathcal{F}\left\{d_{k}^{c a f}\right\}= \begin{cases}\mathcal{F}\left\{d_{k}\right\}(\omega) \cdot W_{k}(\omega) & \text { if } \omega \in\left[-k \frac{B}{2}, k \frac{B}{2}\right], \\ 0 & \text { otherwise }\end{cases}
$$




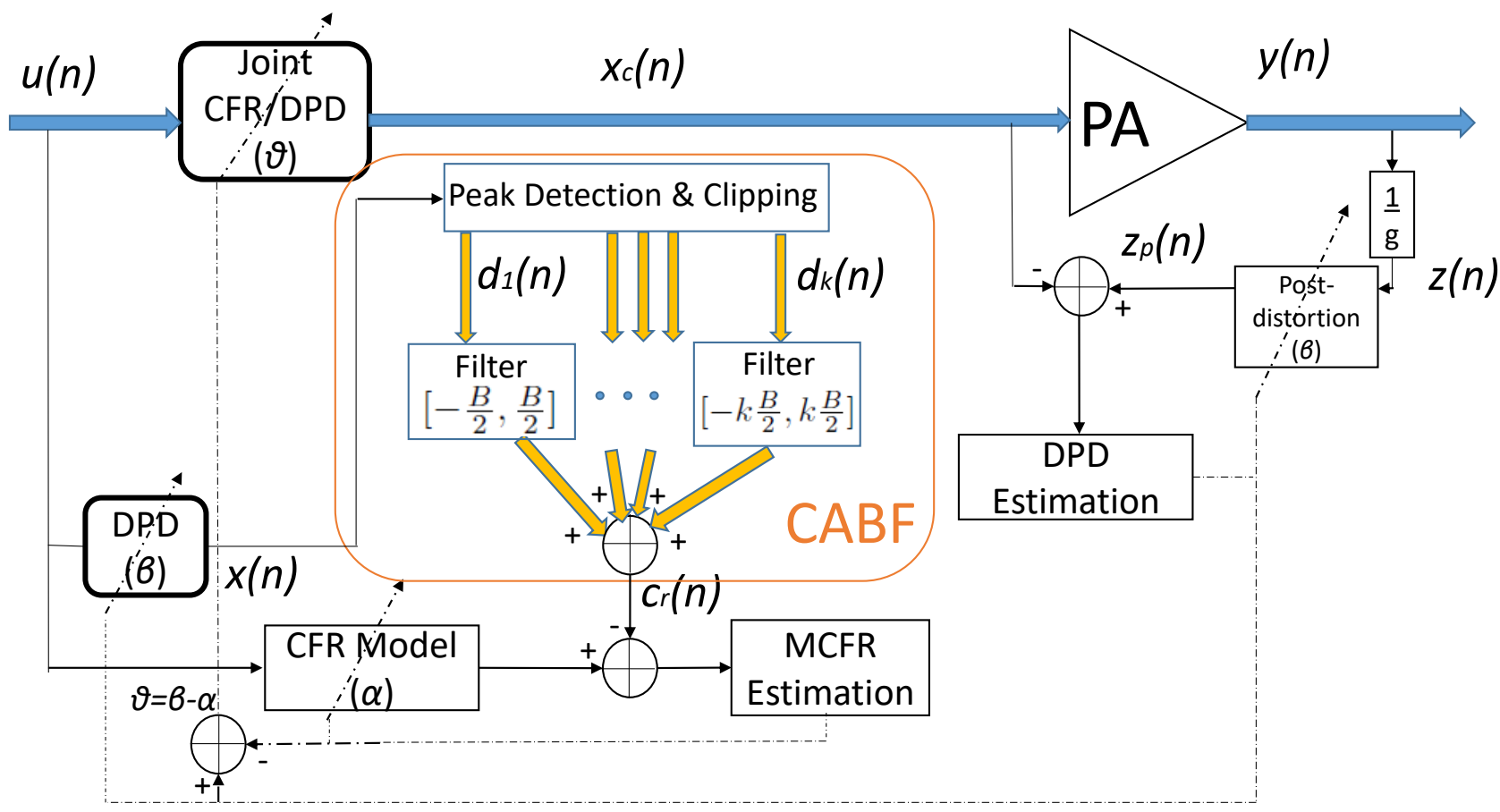

Fig. 3. Proposed joint CFR/DPD structure.

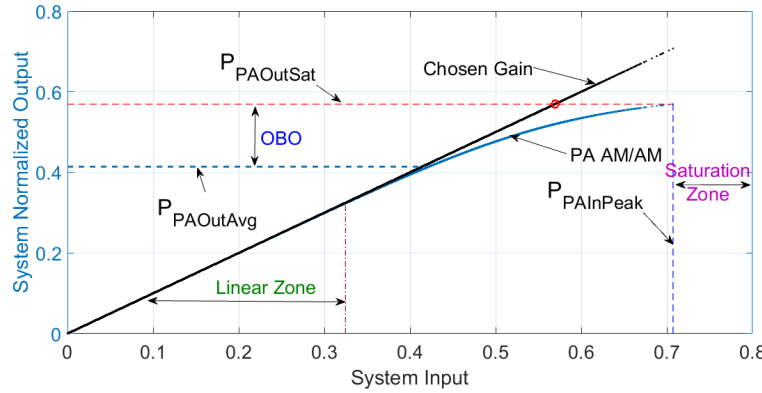

Fig. 4. PA output signal vs PA input signal.

where $W_{k}(\omega)$ is a window centered at frequency 0 with width $\left[-k \frac{B}{2}, k \frac{B}{2}\right]$. The window length is equal to the number of samples of $\mathcal{F}\left\{d_{k}\right\}(\omega)$. Since $g_{k}(n)(k>1)$ occupies a larger band than $g_{1}(n)$, filtering $d_{k}(n)$ increases the errors in bands $\left[-k \frac{B}{2},-\frac{B}{2}\right]$ and $\left[\frac{B}{2}, k \frac{B}{2}\right]$, which deteriorates the ACPR. Hence we propose to apply nonrectangular windows $W_{k}(\omega)$ to shape the filters and to smooth out the error.

As can be done with conventional CAF, we apply $\mathrm{HC}$ on the final signal to ensure no peak exceeding $P$ after repeating the clipping and filtering steps. The reference correction signal is finally computed as

$$
c_{r}^{c a b f}(n)=\sum_{k \in \mathcal{K}} d_{k}^{c a f}(n) .
$$

\section{Choice of Windows}

The choice of windows $W_{k}(\omega)$ has an impact on the linearization performance. To compare different windows based on their intrinsic performance without any influence of the

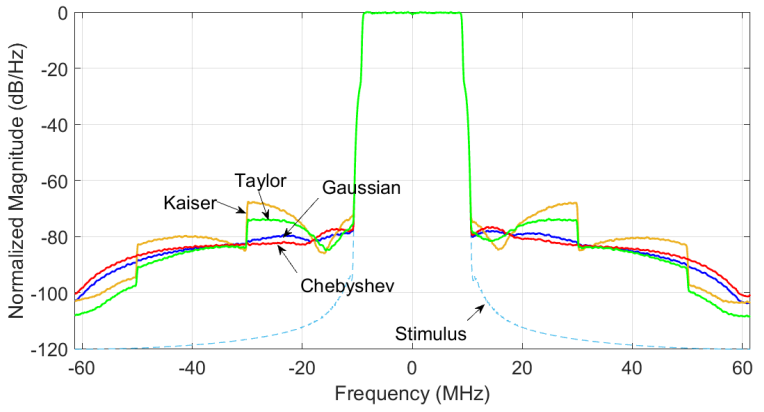

Fig. 5. Test of different windows for CABF.

TABLE I

IMPACTS ON PA LINEARITY WITH DIFFERENT WINDOWS FOR CABF

\begin{tabular}{|l|c|c|c|c|}
\hline \multirow{2}{*}{ Window } & \multicolumn{4}{|c|}{ ACPR $(\mathrm{dBc})$} \\
\cline { 2 - 5 } & L1 & U1 & L2 & U2 \\
\hline Hann & -76.3 & -75.2 & -83.5 & -84.7 \\
\hline Gaussian & -76.7 & -75.9 & -82.1 & -83.0 \\
\hline Hamming & -75.4 & -74.4 & -82.8 & -83.7 \\
\hline Kaiser & -71.3 & -71.3 & -78.6 & -79.0 \\
\hline Blackman & -77.1 & -76.2 & -82.5 & -83.5 \\
\hline Bohman & -77.3 & -76.3 & -82.4 & -83.3 \\
\hline Bartlett & -74.7 & -74.0 & -81.3 & -82.3 \\
\hline Chebyshev & -77.3 & -76.6 & -81.4 & -82.1 \\
\hline Taylor & -74.0 & -73.4 & -83.2 & -83.6 \\
\hline
\end{tabular}

L(U)1(2): First(Second) lower(upper) adjacent channel

MCFR parametric model, we do not estimate the MCFR coefficients but simply pass the clipped reference signal $x_{c r}(n)=$ $x(n)-c_{r}^{c a b f}(n)$ to the PA and evaluate the corresponding PA output spectrum.

In order to test the performance of different windows, a Wiener model PA with $1 \mathrm{~dB}$ compression output power at $25 \mathrm{dBm}$ is used for the simulation on Matlab. A $20 \mathrm{MHz}$ 


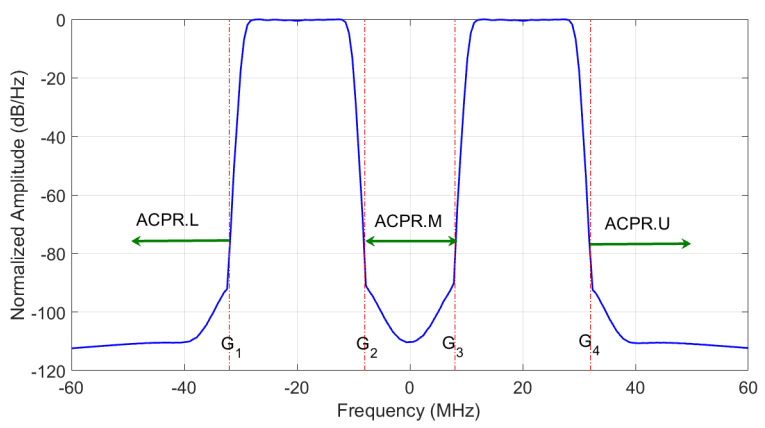

Fig. 6. Notions of bands in case of multi-carrier stimulus.

LTE signal with 90000 samples is used as stimulus. Its PAPR at the $10^{-4}$ probability level is $8 \mathrm{~dB}$. The spectra of the PA output with $8 \mathrm{~dB}$ back-off applied are illustrated in Fig. 5 . The ACPR values are listed in Table I. As a reference, the ACPR value results for the clipped reference signal obtained with conventional $\mathrm{CAF}$ are in $\mathrm{dBc}(-71.5,-71.1)$ for $\mathrm{L} 1 / \mathrm{U} 1$ and $(-72.0,-72.3)$ for $\mathrm{L} 2 / \mathrm{U} 2$. The Chebyshev window with $100 \mathrm{~dB}$ of sidelobe attenuation can reach the best first channel ACPR values (ACPR.L1 and ACPR.U1) and will be used in this paper.

In the next section, we consider the case where the stimulus has multiple carriers.

\section{CABF-based Joint CFR/DPD with Multi-Carrier Stimulus}

When using single-carrier $\mathrm{CABF}$ with the stimulus consisting of multiple carriers, the out-of-band distortion brought by clipping will not be filtered out between channels. Taking the example of a two-carrier signal as Fig. 6, the ACPR needs to be improved not only in lower (ACPR.L) and upper channels (ACPR.U) but also the middle channel (ACPR.M), whose frequency band is $\left[G_{2}, G_{3}\right]$.

We can reduce the values of ACPR.L and ACPR.U using the filter (15) in the previous section. However, in order to filter the middle channel, the windows need to be adapted in the band $\left[G_{2}, G_{3}\right]$.

A $T$-carrier stimulus occupies $T$ frequency bands centered at $f_{c}^{i}$, where $i=1, \cdots, T$, of widths $B_{i}$ and limits $\left[G_{2 i-1}, G_{2 i}\right]$. The distortion on side channels ( $\mathrm{L}$ and $\mathrm{U}$ ) and middle channel (M) are treated separately.

Firstly, for the distortion on side channels, we take the signal as single-carrier of bandwidth $B_{0}=G_{2 T}-G_{1}$ and use the method of (15) with the global window $W_{k}^{0}(\omega)$ defined on $\left[-k \frac{B_{0}}{2}, k \frac{B_{0}}{2}\right]$.

Secondly, for the distortion on middle channels $\left[G_{2 i}, G_{2 i+1}\right]$ where $i=1, \cdots, T-1$, we can first treat them individually as the side channels of each carrier. For the carrier at $f_{c}^{i}$, we create a local window $W_{k}^{i}(\omega)$ in band $\left[f_{c}^{i}-k \frac{B_{i}}{2}, f_{c}^{i}+k \frac{B_{i}}{2}\right]$. When the nonlinearity order $k$ is high enough, the windows for the different carriers may overlap with each other. We take the maximum value among them $\max _{i}\left(W_{k}^{i}(\omega)\right)$ at each overlapped frequency $\omega$. Thus the inner window $\Omega_{k}(\omega)$ in the frequency $\left[G_{1}, G_{2 T}\right]$ is obtained by combining the $W_{k}^{i}(\omega)$ into:

$$
\Omega_{k}(\omega)=\max _{i}\left(W_{k}^{i}(\omega)\right), \omega \in\left[G_{1}, G_{2 T}\right] .
$$

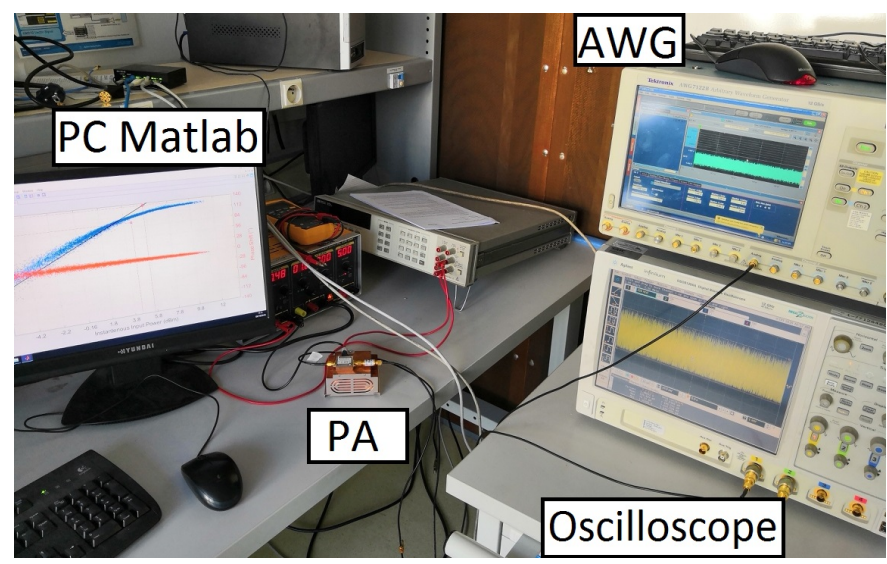

Fig. 7. Test bench for experimental implementation.

We apply this inner window only on the middle channels:

$$
\mathcal{F}\left\{d_{k}^{c a f}\right\}= \begin{cases}\mathcal{F}\left\{d_{k}\right\}(\omega) \cdot W_{k}^{0}(\omega) & \text { if } \omega \in \mathcal{O}, \\ \mathcal{F}\left\{d_{k}\right\}(\omega) \cdot \Omega_{k}(\omega) & \text { if } \omega \in \mathcal{U}, \\ 0 & \text { otherwise }\end{cases}
$$

where $\mathcal{U}$ is the union of the middle channels, and $\mathcal{O}$ is the complementary such that $\mathcal{U} \cup \mathcal{O}=\left[-k \frac{B_{0}}{2}, k \frac{B_{0}}{2}\right]$ :

$$
\begin{aligned}
& \mathcal{U}=\left[G_{2}, G_{3}\right] \cup \ldots \cup\left[G_{2 T-2}, G_{2 T-1}\right], \\
& \mathcal{O}=\left[-k \frac{B}{2}, G_{2}\right] \cup\left[G_{3}, G_{4}\right] \cup \ldots \cup\left[G_{2 T-1}, k \frac{B}{2}\right] .
\end{aligned}
$$

The reference correction signal is then obtained by (16).

\section{EXPERIMENTAL RESULTS}

\section{A. Test bench}

The test bench is illustrated in Fig. 7. Two PAs are used as device under test (DUT) in this section: a HMC409LP4E PA working in 3.3-3.8 GHz for single-carrier test and a TA020060-30-27 PA working in 2-6 GHz for multiple-carrier test.

We generate the modulated signal in the PC Workstation and feed it to the PA through an Arbitrary Waveform Generator (AWG) with $10 \mathrm{GHz}$ sampling frequency.

The PA output signal is captured by an oscilloscope and is fed back to the PC workstation for postdistortion processing. The input and output baseband signals are synchronized in time after down-sampling to $120 \mathrm{MHz}$ for single-carrier stimulus and $600 \mathrm{MHz}$ for multi-carrier stimulus to be used by the identification algorithm.

We feed a segment of 90000 samples signal to the AWG for transmission. The coefficients of the proposed joint CFR/DPD are estimated with 40000 samples in each iteration. The model identification stops after its linearization performance converges. In the implementations of this paper, we keep updating the model coefficients for 4 iterations. The performance is evaluated with the signal of 90000 samples.

\section{B. Running Complexity}

Since the identification of models is required only when the input signal or the PA behavior is changed, here we consider only running complexity [25]. For the conventional 
TABLE II

NUMBER OF FLOPS FOR OPERATIONS

\begin{tabular}{|l|c|l|c|}
\hline Operation & FLOPs & Operation & FLOPs \\
\hline Real Addition & 1 & Real Multiplication & 1 \\
\hline Real Division & 4 & Complex Addition & 2 \\
\hline Complex Multiplication & 6 & $\begin{array}{l}\text { Complex-Real } \\
\text { Multiplication }\end{array}$ & 2 \\
\hline Square-root & $6 \sim 8$ & & \\
\hline
\end{tabular}

CAF-DPD method, we calculate the complexities of the CAF and the DPD separately and their sum is considered as the total complexity of the system. For joint CFR/DPD model, there is only one model which has the same structure than the DPD model. Thus its complexity should be equal to the complexity of the DPD. The only difference that the proposed method exhibits with respect to conventional joint CFR/DPD is regarding model coefficient estimation. Therefore their running complexities are identical.

We estimate the complexity of each method by the number of FLOPs (floating-point operation). Table II gives the number of FLOPs of each operation according to [25]. Since different algorithms are available for the square-root operation, the number of FLOPs is around 6 8. We take the average 7 FLOPs in this paper.

1) Joint $C F R / D P D$ complexity estimation: The joint CFR/DPD is implemented with a GMP model in this paper. According to [25], the running complexity of a GMP model for an $N$-sample signal can be expressed as

$$
F_{G M P}=(8 R-2) N
$$

where $R$ is the number of model coefficients.

2) CAF complexity estimation: We clip and filter the signal iteratively using the CAF approach. By detecting the peaks, one complex modulus is computed for each sample. In order to reduce the number of processed samples, the peak windowing technique is taken in place of a low-pass filter. A Gaussian window is applied on $S$ samples centered at the clipped peak. Two complex subtractions (4 FLOPs) on each sample are implemented before and after filtering. The number of peaks detected decreases as long as the number of CAF iteration increases. If we denote the average number of peaks per iteration as $\bar{N}_{p}$, the number of FLOPs for an $N$-sample signal at each iteration is $14 N+\bar{N}_{p}(6+2 S)$.

If there are still $N_{p}^{\prime}$ peaks remained after $I_{m}$ iterations for CAF, we hard clip them. The total number of FLOPs is then

$$
F_{C A F}=I_{m}\left[14 N+\bar{N}_{p}(6+2 S)\right]+\left(10 N+6 N_{p}^{\prime}\right) .
$$

In this paper we set the Gaussian window width $S=100$ and CAF iteration number $I_{m}=10$.

\section{Measurement result with single-carrier stimulus}

In this section we use as stimulus a $20 \mathrm{MHz}$ LTE signal with PAPR equal to $8 \mathrm{~dB}$ at $3.5 \mathrm{GHz}$ carrier frequency. The DUT is a HMC409LP4E PA fabricated by Analog Devices. Its nominal gain at $3.5 \mathrm{GHz}$ frequency is $31 \mathrm{~dB}$ and the output power at $1 \mathrm{~dB}$ compression point is $32.5 \mathrm{dBm}$. The supply voltage is $5 \mathrm{~V}$. The proposed joint CFR/DPD is tested on test bench and is compared with the conventional CAF-DPD method and the conventional joint CFR/DPD method.

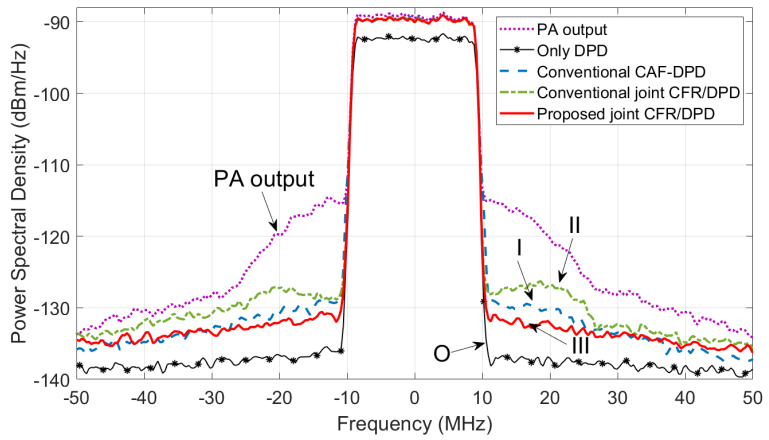

O: Only DPD without CFR; I: Conventional CAF-DPD method; II: Conventional joint CFR/DPD method; III: Proposed joint CFR/DPD method

Fig. 8. PA output spectra for various system configurations: without DPD, with DPD only, and with DPD and CFR using methods I, II, III for singlecarrier $20 \mathrm{MHz}$ LTE.

TABLE III

COMPARISON OF DIFFERENT METHODS WITH SINGLE-CARRIER $20 \mathrm{MHZ}$ LTE STIMULUS

\begin{tabular}{|l|c|c|c|c|c|}
\hline \multicolumn{2}{|c|}{} & O & I & II & III \\
\hline \multicolumn{2}{|c|}{$P_{\text {out }}(\mathrm{dBm})$} & 24.2 & 26.8 & 26.6 & 26.6 \\
\hline \multicolumn{2}{|c|}{$I_{\text {supply }(\mathrm{A})}$} & 0.63 & 0.68 & 0.68 & 0.68 \\
\hline \multicolumn{2}{|c|}{ PAE (\%) } & 8.4 & 14.1 & 13.4 & 13.4 \\
\hline \multirow{3}{*}{$\begin{array}{l}\text { ACPR } \\
(\mathrm{dBc})\end{array}$} & $\mathrm{L} 1$ & -45.0 & -40.9 & -38.6 & -42.7 \\
\cline { 2 - 6 } & $\mathrm{U} 1$ & -45.7 & -41.0 & -38.4 & -43.3 \\
\cline { 2 - 6 } & $\mathrm{L} 2$ & -46.3 & -45.0 & -42.8 & -45.0 \\
\cline { 2 - 6 } & $\mathrm{U} 2$ & -46.8 & -46.0 & -44.3 & -45.8 \\
\hline \multicolumn{2}{|c|}{ EVM (\%) } & 2.4 & 3.6 & 4.5 & 4.7 \\
\hline \multicolumn{2}{|c|}{ Complexity (flops) } & $1.49 e^{7}$ & $2.87 e^{7}$ & $1.49 e^{7}$ & $1.49 e^{7}$ \\
\hline
\end{tabular}

O: Only DPD without CFR

I: Conventional CAF-DPD method

II: Conventional joint CFR/DPD method

III: Proposed joint CFR/DPD method

With the PA input and output signals, the optimal DPD model structure determined by the algorithm in [21] is

$$
\begin{aligned}
\mathcal{K}_{a} & =2, \mathcal{L}_{a}=4 \\
\mathcal{K}_{b} & =1, \mathcal{L}_{b}=1, \mathcal{M}_{b}=1 \\
\mathcal{K}_{c} & =6, \mathcal{L}_{c}=2, \mathcal{M}_{c}=1 .
\end{aligned}
$$

For the sake of simplicity, we use the same DPD model structure for the joint CFR/DPD model.

The PA output spectra linearized with different methods are illustrated in Fig. 8. The pink dotted curve is the PA output without DPD linearization. The black star curve stands for the PA output linearized by only DPD (method O). The blue dashed curve represents the conventional CAF-DPD method (method I). The green dashed-dotted curve represents the conventional CFR/DPD method (method II). The red curve represents the proposed CABF-based CFR/DPD method (method III).

The corresponding ACPR, EVM values, complexities, the currents of supply ( $\left.I_{\text {supply }}\right)$ and PA power added efficiencies (PAE) are given in Table III. The complexities are estimated with $N=90000$ samples.

The EVM values of all approaches are less than 5\%. The result of method O (only DPD without CFR) is given as a reference.

The operating point of method $\mathrm{O}$ is limited by the PAPR 


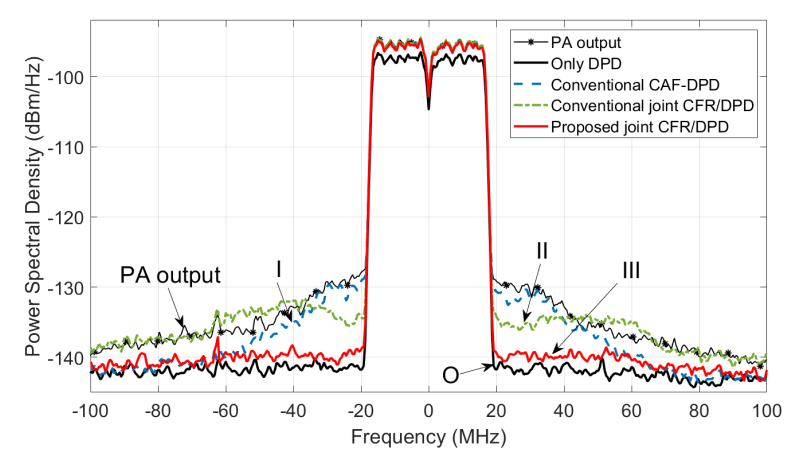

O: Only DPD without CFR; I: Conventional CAF-DPD method; II: Conventional joint CFR/DPD method; III: Proposed joint CFR/DPD method

Fig. 9. PA output spectra for various system configurations: without DPD, with DPD only, and with DPD and CFR using methods I, II, III for singlecarrier $40 \mathrm{MHz}$ WLAN signal.

TABLE IV

COMPARISON OF DIFFERENT METHODS WITH SINGLE-CARRIER $40 \mathrm{MHZ}$ WLAN STIMULUS

\begin{tabular}{|l|c|c|c|c|c|}
\hline \multicolumn{2}{|c|}{} & O & I & II & III \\
\hline \multicolumn{2}{|c|}{$P_{\text {out }}(\mathrm{dBm})$} & 20.5 & 22.6 & 22.7 & 22.4 \\
\hline \multicolumn{2}{|c|}{$I_{\text {supply }}(\mathrm{A})$} & 0.63 & 0.63 & 0.63 & 0.63 \\
\hline \multicolumn{2}{|c|}{ PAE (\%) } & 3.6 & 5.6 & 5.9 & 5.5 \\
\hline \multirow{3}{*}{$\begin{array}{l}\text { ACPR } \\
(\mathrm{dBc})\end{array}$} & L1 & -44.2 & -35.9 & -37.5 & -43.3 \\
\cline { 2 - 6 } & $\mathrm{U} 1$ & -44.6 & -35.8 & -39.0 & -43.4 \\
\cline { 2 - 6 } & $\mathrm{L} 2$ & -44.8 & -42.1 & -42.1 & -44.5 \\
\cline { 2 - 6 } & $\mathrm{U} 2$ & -45.9 & -43.3 & -44.1 & -45.6 \\
\hline \multicolumn{2}{|c|}{ EVM (\%) } & 3.5 & 2.2 & 4.6 & 2.4 \\
\hline \multicolumn{2}{|c|}{ Complexity (flops) } & $2.34 e^{7}$ & $7.29 e^{7}$ & $2.34 e^{7}$ & $2.34 e^{7}$ \\
\hline
\end{tabular}

O: Only DPD without CFR

I: Conventional CAF-DPD method

II: Conventional joint CFR/DPD method

III: Proposed joint CFR/DPD method

of the stimulus. Compared with method O, the other methods with CFR which reduces the PAPR of around $2.5 \mathrm{~dB}$ can improve the PAE from $8.4 \%$ to at least $13.4 \%$. These results confirm the benefit of the CFR techniques on the power efficiency improvement.

Compared with method I, method II has lower complexity but worse linearization performance. Compared with method I and II, our proposed joint CFR/DPD (method III) has overall better ACPR values and complexity. We save $48 \%$ of the complexity from the conventional CAF-DPD method. The ACPR values of the proposed joint CFR/DPD on the first adjacent channels have an advantage beyond the conventional joint CFR/DPD of up to $5 \mathrm{~dB}$.

We also test these approaches using a $40 \mathrm{MHz}$ Wireless Local Area Network (WLAN) signal as the stimulus. The PAPR of the stimulus is $12 \mathrm{~dB}$. The optimal DPD model structure becomes

$$
\begin{aligned}
\mathcal{K}_{a} & =1, \mathcal{L}_{a}=11 \\
\mathcal{K}_{b} & =3, \mathcal{L}_{b}=2, \mathcal{M}_{b}=1 \\
\mathcal{K}_{c} & =8, \mathcal{L}_{c}=2, \mathcal{M}_{c}=1 .
\end{aligned}
$$

The PA output spectra obtained for different CFR and DPD approaches are illustrated in Fig. 9. The numerical results are listed in Table IV.

The PA output power is increased by $2 \mathrm{~dB}$ with combined

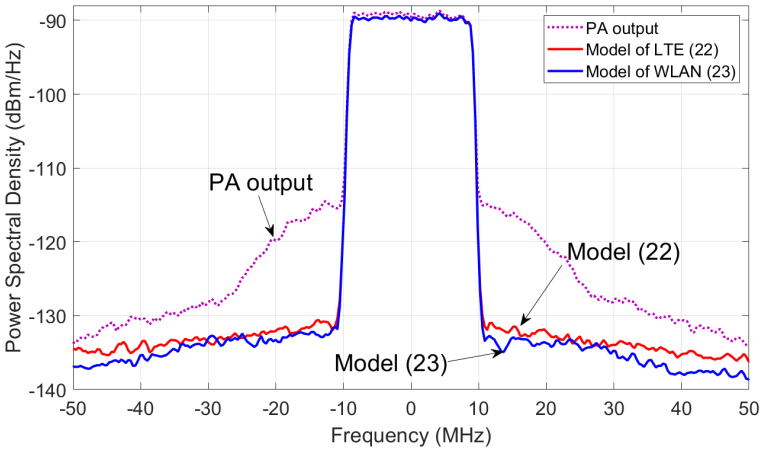

Fig. 10. PA output spectra with proposed joint CFR/DPD method using different models for single-carrier $20 \mathrm{MHz}$ LTE signal.

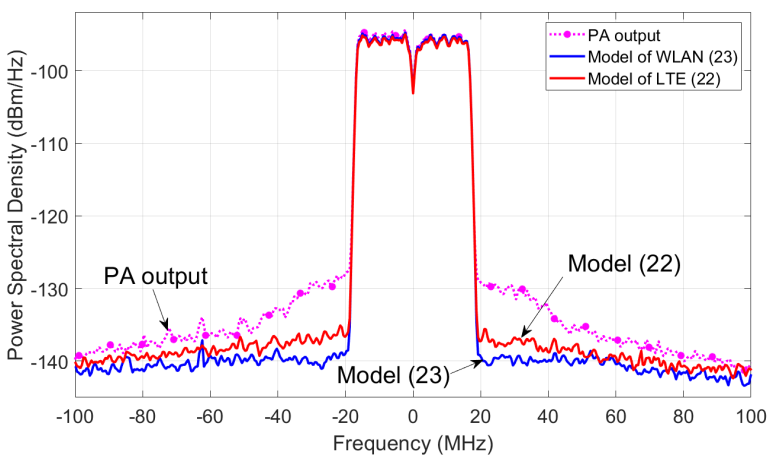

Fig. 11. PA output spectra with proposed joint CFR/DPD method using different models for single-carrier $40 \mathrm{MHz}$ WLAN signal.

TABLE V

COMPARISON OF DIFFERENT MODELS

\begin{tabular}{|l|c|c|c|c|c|}
\hline \multicolumn{2}{|c|}{ Signal } & \multicolumn{2}{c|}{ LTE } & \multicolumn{2}{c|}{ WLAN } \\
\hline \multicolumn{2}{|c|}{ Model } & $(22)$ & $(23)$ & $(22)$ & $(23)$ \\
\hline \multirow{3}{*}{$\begin{array}{l}\text { ACPR } \\
(\mathrm{dBc})\end{array}$} & L1 & -42.7 & -42.9 & -40.7 & -43.3 \\
\cline { 2 - 6 } & $\mathrm{U} 1$ & -43.3 & -43.8 & -41.4 & -43.4 \\
\cline { 2 - 6 } & L2 & -45.0 & -46.0 & -42.8 & -44.5 \\
\cline { 2 - 6 } & $\mathrm{U} 2$ & -45.8 & -47.4 & -44.1 & -45.6 \\
\hline \multicolumn{2}{|c|}{ EVM (\%) } & 4.7 & 3.6 & 3.0 & 2.4 \\
\hline \multicolumn{2}{|c|}{ Complexity (flops) } & $1.49 e^{7}$ & $2.34 e^{7}$ & $1.49 e^{7}$ & $2.34 e^{7}$ \\
\hline
\end{tabular}

CFR and DPD methods (Method I-III), which renders an improvement of $56 \%$ on the PA efficiency. Our proposed method (Method III) has very good performance on PA linearization and complexity compared with Method I and II. The EVM value is kept under $5 \%$.

These experimental results validate the robustness of the proposed CABF-based joint CFR/DPD. Since one aim of CFR is to increase the PA power efficiency by pushing its operating point towards the saturation zone, the PA linearity should deteriorate. At the same operating point, our proposed method reaches better ACPR values compared with the conventional methods. In other words, it provides a larger range of operating point choice since it gives larger margin on ACPR.

The performance of the proposed joint CFR/DPD is also evaluated with different models (22) and (23). The PA output spectra of these tests are illustrated in Fig. 10 and Fig. 11 using LTE and WLAN signal respectively. The corresponding 


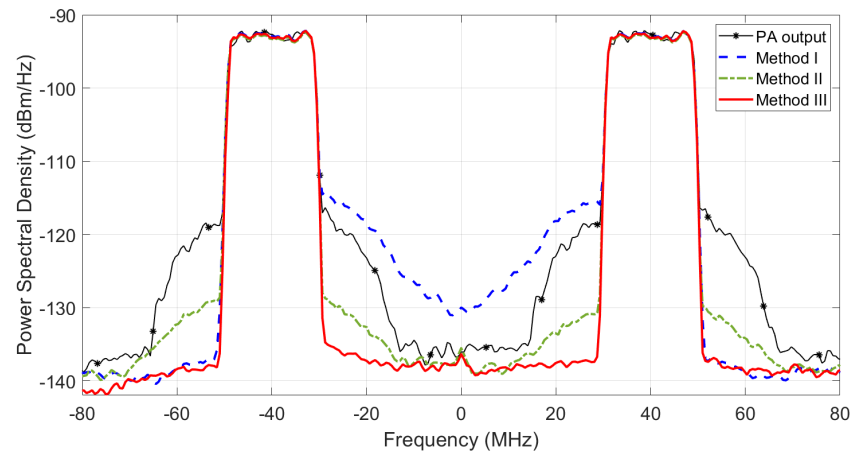

I: Conventional CAF-DPD method; II: Conventional joint CFR/DPD method; III: Proposed joint CFR/DPD method

Fig. 12. PA output spectra for various system configurations: without DPD, with DPD and CFR using methods I, II, III for 2-carrier $20 \mathrm{MHz}$ LTE.

TABLE VI

COMPARISON OF DIFFERENT METHODS WITH TWO-CARRIER STIMULUS

\begin{tabular}{|l|c|c|c|c|}
\hline \multicolumn{2}{|c|}{} & I & II & III \\
\hline \multirow{3}{*}{$\begin{array}{c}\text { ACPR } \\
(\mathrm{dBc})\end{array}$} & L & -45.0 & -39.2 & -44.9 \\
\cline { 2 - 5 } & M.L & -26.2 & -39.6 & -43.1 \\
\cline { 2 - 5 } & M.U & -26.2 & -40.1 & -43.9 \\
\cline { 2 - 5 } & $\mathrm{U}$ & -44.9 & -40.8 & -44.4 \\
\hline \multicolumn{2}{|c|}{ EVM (\%) } & 3.2 & 6.8 & 3.7 \\
\hline \multicolumn{2}{|c|}{ Complexity (flops) } & $2.56 e^{7}$ & $7.02 e^{6}$ & $7.02 e^{6}$ \\
\hline
\end{tabular}

I: Conventional CAF-DPD method

II: Conventional joint CFR/DPD method

III: Proposed joint CFR/DPD method

ACPR and EVM values and complexities are listed in Table V.

When using LTE signal as the stimulus, model (23) keeps a good linearization performance. The complexity of (23) is higher, but comparing with the conventional CFR-DPD method in Table III, we can still save $23 \%$ of complexity. When using WLAN signal as the stimulus, model (22) degrades over $2 \mathrm{~dB}$ on ACPR with a much lower complexity.

\section{Measurement result with 2-Carrier stimulus}

We test the proposed multi-carrier CABF-based joint CFR/DPD in this section. The DUT in the test bench is a TA020-060-30-27 PA fabricated by Transcom. Its nominal gain is $30 \mathrm{~dB}$ and the output power at $1 \mathrm{~dB}$ gain compression is $27 \mathrm{dBm}$. A 2-carrier LTE signal is used as the stimulus with $10 \mathrm{~dB}$ PAPR. The lower band carrier frequency is $2100 \mathrm{MHz}$ and the upper band carrier frequency is $2180 \mathrm{MHz}$. These two components have both $20 \mathrm{MHz}$ bandwidth. We use a GMP model for the DPD with $\mathcal{K}_{a}=5, \mathcal{L}_{a}=2$ and $\mathcal{K}_{b}=\mathcal{K}_{c}=0$, which corresponds to an MP model.

The PA output spectra linearized with different methods are illustrated in Fig. 12. The black star curve gives the PA output without DPD linearization. The blue dashed curve illustrates the performance of the conventional CAF-DPD method (method I). The green dashed-dotted curve results from the conventional CFR/DPD method (method II). The red curve is the spectrum of the proposed CABF-based CFR/DPD method (method III).
The corresponding ACPR, EVM values, complexities are given in Table VI. Since the advantage on PA efficiency of applying CFR has been addressed in last section, here we compare only the results of the methods with CFR at the same PA operating point. For ACPR values, we estimate band L $(-70 \mathrm{MHz}$ to $-50 \mathrm{MHz})$ and M.L $(-30 \mathrm{MHz}$ to $-10 \mathrm{MHz})$ for the lower band carrier, band M.U (10 MHz to $30 \mathrm{MHz}$ ) and $\mathrm{U}(50 \mathrm{MHz}$ to $70 \mathrm{MHz}$ ) for the upper band carrier. The complexities are estimated with $N=90000$ samples.

The EVM value of our proposed method is kept less than $4 \%$. By using the joint CFR/DPD structure, methods II and III save $72 \%$ of the complexity from the conventional CAFDPD method. Compared with method II, our proposed CABFbased method exhibits better ACPR performance and the improvements in the middle band (M.L/U) and lower/upper band $(\mathrm{L} / \mathrm{U})$ are around $4 \mathrm{~dB}$.

The comparison against the conventional methods in Table VI shows that our proposed CABF-based joint CFR/DPD achieves good performance on both the sidebands and the middle band with lower complexity.

\section{CONClusion}

This paper explores a CABF-based joint CFR/DPD approach when the system input signal can have multiple carriers. Experimental results with two different PAs show that the proposed approach takes full advantage of the joint CFR/DPD paradigm in terms of complexity reduction while simultaneously providing better linearization performances than conventional joint CFR/DPD methods in both single-carrier and multi-carrier cases. Concerning the choice of windows for the bank filtering, Chebychev windows are suggested since they give the most relevant performance in terms of ACPR.

\section{REFERENCES}

[1] L. Guan and A. Zhu, "Green communications: Digital predistortion for wideband rf power amplifiers," IEEE Microw. Mag., vol. 15, no. 7, pp. 84-99, Nov 2014.

[2] F. Raab, "Efficiency of doherty rf power-amplifier systems," IEEE Trans. Broadcast., vol. BC-33, no. 3, pp. 77-83, Sept. 1987.

[3] A. Katz, J. Wood, and D. Chokola, "The evolution of pa linearization: From classic feedforward and feedback through analog and digital predistortion," IEEE Microw. Mag., vol. 17, no. 2, pp. 32-40, Feb 2016.

[4] J. Kim and K. Konstantinou, "Digital predistortion of wideband signals based on power amplifier model with memory," Electronics Letters, vol. 37, no. 23, pp. 1417-1418, Nov 2001.

[5] L. Ding, G. Zhou, D. Morgan, Z. Ma, J. Kenney, J. Kim, and C. Giardina, "A robust digital baseband predistorter constructed using memory polynomials," IEEE Trans. Commun., vol. 52, no. 1, pp. 159-165, Jan. 2004.

[6] D. Morgan, Z. Ma, J. Kim, M. Zierdt, and J. Pastalan, "A generalized memory polynomial model for digital predistortion of rf power amplifiers," IEEE Trans. Signal Process., vol. 54, no. 10, pp. 3852-3860, Oct. 2006.

[7] A. Zhu, J. Pedro, and T. Brazil, "Dynamic deviation reduction-based volterra behavioral modeling of rf power amplifiers," IEEE Trans. Microw. Theory Techn., vol. 54, no. 12, pp. 4323-4332, Dec 2006.

[8] F. Mkadem, M. C. Fares, S. Boumaiza, and J. Wood, "Complexityreduced volterra series model for power amplifier digital predistortion," Analog Integrated Circuits and Signal Processing, vol. 79, no. 2, pp. 331-343, 2014

[9] A. Zhu, "Decomposed vector rotation-based behavioral modeling for digital predistortion 1t;newline/ gt; of rf power amplifiers," IEEE Trans. Microw. Theory Techn., vol. 63, no. 2, pp. 737-744, Feb 2015. 
[10] T. Liu, S. Boumaiza, and F. Ghannouchi, "Augmented hammerstein predistorter for linearization of broad-band wireless transmitters," IEEE Trans. Microw. Theory Techn., vol. 54, no. 4, pp. 1340-1349, Jun 2006.

[11] S. Chen, "An efficient predistorter design for compensating nonlinear memory high power amplifiers," IEEE Trans. Broadcast., vol. 57, no. 4, pp. $856-865$, dec. 2011.

[12] S. Wang, M. Abi Hussein, O. Venard, and G. Baudoin, "Optimal sizing of two-stage cascaded sparse memory polynomial model for high power amplifiers linearization," IEEE Trans. Microw. Theory Techn., vol. 66, no. 9, pp. 3958-3965, Sep 2018.

[13] J. Wood, Behavioral Modeling and Linearization of RF Power Amplifiers:, ser. Artech House Microwave Library. Artech House, 2014.

[14] R. N. Braithwaite, "A combined approach to digital predistortion and crest factor reduction for the linearization of an rf power amplifier," IEEE Trans. Microw. Theory Techn., vol. 61, no. 1, pp. 291-302, Jan 2013.

[15] A. Mbaye, G. Baudoin, A. Gouba, Y. Louet, and M. Villegas, "Combining crest factor reduction and digital predistortion with automatic determination of the necessary crest factor reduction gain," in 2014 44th European Microwave Conference, Oct 2014, pp. 837-840.

[16] X. Chen, W. Chen, F. Huang, F. M. Ghannouchi, Z. Feng, and Y. Liu, "Systematic crest factor reduction and efficiency enhancement of dualband power amplifier based transmitters," IEEE Trans. Broadcast., vol. 63, no. 1, pp. 111-122, Mar 2017.

[17] S. Wang, M. Roger, and C. Lelandais-Perrault, "Impacts of crest factor reduction and digital predistortion on linearity and power efficiency of power amplifiers," IEEE Trans. Circuits Syst. II, Exp. Briefs, vol. 66 , no. 3, pp. 407-411, Mar 2019.

[18] H. Enzinger, K. Freiberger, and C. Vogel, "Competitive linearity for envelope tracking: Dual-band crest factor reduction and 2d-vectorswitched digital predistortion," IEEE Microw. Mag., vol. 19, no. 1, pp. 69-77, Jan 2018.

[19] M. V. Amiri, M. Helaoui, N. Boulejfen, and F. M. Ghannouchi, "Optimized spectrum constrained crest factor reduction technique using polynomials," IEEE Trans. Commun., vol. 63, no. 7, pp. 2555-2564, Jul 2015.

[20] A. Mammela and A. Anttonen, "Why will computing power need particular attention in future wireless devices?" IEEE Circuits Syst. Mag., vol. 17, no. 1, pp. 12-26, Firstquarter 2017.

[21] S. Wang, M. Abi Hussein, O. Venard, and G. Baudoin, "A novel algorithm for determining the structure of digital predistortion models," IEEE Trans. Veh. Technol, vol. 67, no. 8, pp. 7326-7340, Aug 2018.

[22] M. Abi Hussein, V. Bohara, and O. Venard, "On the system level convergence of ila and dla for digital predistortion," in Wireless Communication Systems (ISWCS), 2012 International Symposium on, Aug 2012, pp. 870-874.

[23] S. Wang, M. Roger, and C. Lelandais-Perrault, "Clipping-and-bankfiltering technique in joint crest factor reduction and digital predistortion for power amplifiers," in 2018 Asia-Pacific Microwave Conference (APMC), Nov 2018, pp. 768-770.

[24] K. Anoh, C. Tanriover, and B. Adebisi, "On the optimization of iterative clipping and filtering for papr reduction in ofdm systems," IEEE Access, vol. 5, pp. 12004-12013, 2017.

[25] A. S. Tehrani, H. Cao, S. Afsardoost, T. Eriksson, M. Isaksson, and C. Fager, "A comparative analysis of the complexity/accuracy tradeoff in power amplifier behavioral models," IEEE Trans. Microw. Theory Techn., vol. 58, no. 6, pp. 1510-1520, Jun 2010.

[26] C. Kantana, O. Venard, and G. Baudoin, "Comparison of gmp and dvr models," in 2018 International Workshop on Integrated Nonlinear Microwave and Millimetre-wave Circuits (INMMIC), Jul 2018, pp. 1-3.

[27] S. Wang, M. Abi Hussein, O. Venard, and G. Baudoin, "Impact of the normalization gain of digital predistortion on linearization performance and power added efficiency of the linearized power amplifier," in 2017 12th European Microwave Integrated Circuits Conference (EuMIC), Oct 2017, pp. 310-313.

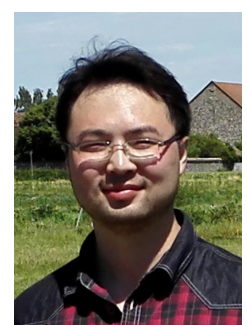

Siqi Wang received the B.S. degree from Huazhong University of Science and Technology, Wuhan, China in 2012, the M.S. degree from the University of Paris-Sud, Orsay, France in 2014, and the Ph.D. degree from the University of Paris-Est Marne La Vallée, Champs sur Marne, France, in 2018. He is currently a post-doc fellow with GeePs, Centralesupélec. His research interests include wireless communications, digital predistortion, energy efficiency optimization for wireless communication systems.

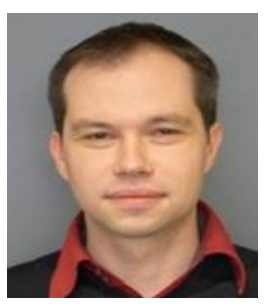

Morgan Roger graduated from the Ecole Spéciale des Travaux Publics in Paris in 2002, with an emphasis on mechanics, electrical engineering and electronics. The same year, he joined the Department of Electronic Systems of CentraleSupélec in Gif-surYvette to pursue his $\mathrm{Ph} . \mathrm{D}$. in signal processing. $\mathrm{He}$ received it from the Paris-Sud University (Orsay) in 2007 and is now an associate professor of CentraleSupélec, affiliated with the GeePs laboratory. His current research interests include data converters and signal processing methods for electronic systems with an emphasis on power amplifier linearization. He is an IEEE member.

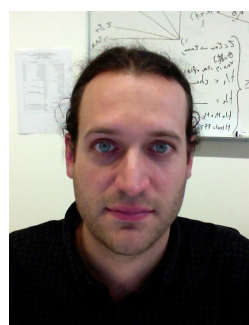

Julien Sarrazin received his Engineering diploma/Master of Research, and $\mathrm{PhD}$ degrees from the University of Nantes in France, in 2005 and 2008 respectively. In 2009 and 2010, he worked at the BK Birla Institute of Technology of Pilani, in India, where he was in charge of telecommunication-related teaching. In 2011 and 2012, he was a research engineer at Telecom ParisTech in Paris. Since September 2012, he is an Associate Professor at Sorbonne Université (formerly University of Pierre and Marie Curie) in Paris, where he is currently working in the Electronics and Electromagnetism Lab (L2E) in the field of Spatial Data Focusing and localization. His research interests also include antenna design, channel modeling for Body Area Networks. He is an IEEE member and reviewer and has published over 90 technical journals, patents, book chapters, and conference papers.

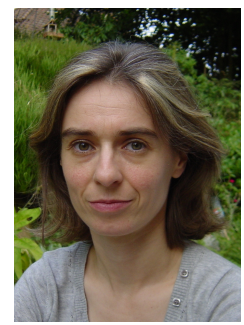

Caroline Lelandais-Perrault received in 1995 the diploma from the Ecole Supérieure d?Electricité (Supélec), one of the French Grandes Ecoles. She worked six years as a development engineer and project manager at IN-SNEC (Zodiac Data Systems), a company specialized in telemetry equipments for satellites and Ariane rocket. In 2001, she joined the Electronic System Department at Supélec and received her PhD in 2006 from Université Paris XI. She is currently Associate Professor in the Dep. of Electronic Systems of CentraleSupélec at Université Paris-Saclay. With GeePs lab. (UMR CNRS 8507), her research activities concern wide-band and versatile and also high resolution analog-to-digital conversion and focus especially on calibration techniques of that systems. 